\section{Reversible metronidazole neurotoxicity in 12 dogs}

\section{Anna Tauro', Elsa Beltran², Colin Driver', Clare Rusbridge $\mathrm{e}^{1,3}$}

\author{
1 Fitzpatrick Referrals, Eashing, UK \\ 2 The Royal Veterinary College, London, UK \\ 3 University of Surrey, Guildford, UK
}

Metronidazole is an antibacterial and antiprotozoal not approved for veterinary use but commonly used to treat systemic and enteric obligate anaerobic bacterial and some protozoal infections. It induces loss of helical structure and strand breakage in bacterial and protozoal DNA and inhibits nucleic acid synthesis resulting in cell death. It has a primarily hepatic metabolism via several pathways. It is lipophilic and rapidly distributed to most body tissues and fluids, including the CNS. Neurotoxicity has been described in dogs receiving $>65 \mathrm{mg} / \mathrm{kg} /$ day. Neurotoxicity may also occur following chronic therapy using dosages as low as $30 \mathrm{mg} / \mathrm{kg} /$ day. The mechanism is not known, however it may be related to modulation of the inhibitory neurotransmitter gammaaminobutyric acid (GABA) receptor within the cerebellar and vestibular systems.

A retrospective cohort study was undertaken to define features related to metronidazole neurotoxicity. Medical records between 2005 and 2015 were reviewed and 12 dogs where identified. Breeds represented were German shepherd dogs (5), Labrador retrievers (2), Labrador crossed Mastiff, Border collie, Soft coated wheaten terrier, Great
Dane and Shih-Tzu.

Mean age of presentation: 7years (range 2-12 years); average of treatment duration: 57days (range 10-150 days); average treatment dose: $22 \mathrm{mg} / \mathrm{kg} / \mathrm{bid}$; median treatment dose: $20 \mathrm{mg} / \mathrm{kg} / \mathrm{bid}$ (range $15-40 \mathrm{mg} / \mathrm{kg} / \mathrm{bid}$ ). Neurotoxicity signs included pathological nystagmus (92\%), vestibulocerebellar ataxia and wide based stance (67\%), non-ambulatory tetraparesis (42\%), hyper-reflexia (25\%), visual\&auditory hyperesthesia (25\%), intentional tremor (17\%), absent menace response (8\%) and altered mentation (8\%). Haematology, serum biochemistry, electrolytes, serology for Toxoplasma gondii \& Neospora caninum, TLI \& folate \& cobalamine serum concentration and cerebrospinal fluid analysis were unremarkable. On MRI one dog had bilateral symmetrical hypertintensity in T2W \& FLAIR images within the dentate nuclei similar to the MRI appearance of this disease in humans. One case with exocrine pancreatic insufficiency that presented with vestibulocerebellar signs and peripheral neuropathy also had hypocobalaminemia. Ach- $R$ antibody test was negative in this case. Dogs improved rapidly within 6 days (range 1-14 days) once the metronidazole was discontinued and diazepam instituted (in 8/12 cases), with the exception of the hypocobalaminemic case, which remained mildly ataxic and tetraparetic 4 weeks later. A diagnosis of metronidazole toxicity was made on the basis of physical examination, exclusion of other disorders and resolution of clinical signs upon discontinuation of the drug.

Metronidazole neurotoxicity may occur following short course ( 2 weeks) of the recommended dose $(\sim 20 \mathrm{mg} / \mathrm{Kg} /$ bid). We therefore suggest caution when administering metronidazole at doses above $40 \mathrm{mg} / \mathrm{kg} /$ day.

\section{Clinical presentation and outcome of dogs treated medically for thoracolumbar anulus fibrosus protrusion}

\section{Abbe Crawford, Steven De Decker}

Royal Veterinary College, London, UK

Intervertebral disc disease is a common spinal disorder. However, the clinical characteristics of dogs with thoracolumbar anulus fibrosus protrusions (AFPs) have only sporadically been reported. Several surgical techniques are described, however little is known about medical management.

A retrospective study was performed to identify risk factors for and associated outcome of dogs treated medically for thoracolumbar AFP. This was done by comparing the clinical presentation of dogs undergoing medical or surgical treatment and evaluating outcome after medical management. Variables compared between medically and surgically treated dogs included signalment, duration and severity of clinical signs, response to medical treatment prior to referral, presence of neurological deficits, presence of concurrent disease, presence of single or multiple sites of AFP, presence of intraparenchymal signal intensity changes and degree of spinal cord compression on magnetic resonance imaging (MRI). Medical treatment consisted of restricted exercise and analgesic medication. Surgical treatment consisted of decompressive surgery (hemilaminectomy or partial corpectomy). Outcome for medically treated dogs was obtained by a standardized questionnaire and review of referring veterinarians' clinical records.

84 dogs receiving medical $(n=31)$ or surgical $(n=53)$ treatment were included. Significantly more medically treated dogs had received successful medical treatment prior to referral and had concurrent disease, while significantly more surgically treated dogs had neurological deficits, were non-ambulatory at the time of presentation and had greater spinal cord compression on MRI. No other assessed variables were found to be significantly different between the 2 treatment groups. Outcome was available for 27 of the 31 medically managed dogs; 11 (40.7\%) improved, 7 (25.9\%) remained stable, and 9 (33.3\%) deteriorated. Of these 9 dogs, 6 (66.7\%) subsequently underwent surgical treatment. The interval from diagnosis to surgical treatment ranged from 6 to 120 days (median 59.5 days). Surgery in these 6 dogs was without complications, and all dogs showed a clinical improvement postoperatively. The median survival time for dogs receiving medical treatment only was 568 days. Ten dogs showed neurological deterioration after initially improving or stabilizing with medical treatment, and were ultimately euthanised due to progression of spinal cord disease.

In conclusion, the clinical presentation differed between dogs managed medically or surgically for thoracolumbar AFP. Medical management can provide a fair prognosis for dogs with thoracolumbar AFP. 